\title{
Higher rectal temperatures in co-sleeping infants
}

\author{
C S Tuffnell, S A Petersen, M P Wailoo
}

\begin{abstract}
The effect on deep body temperature of infants co-sleeping (with either or both parents) is investigated in this case control study. Overnight continuous recordings of rectal temperature were made from 34 babies co-sleeping with one or both parents throughout the night and 34 infants matched for age, feeding regimen, parental smoking, thermal environment, sleeping position, and sex who slept alone. The co-sleeping infants had significantly higher rectal temperatures from two hours after bedtime, when the initial fall in sleeping body temperature was complete. The mean rectal temperature of co-sleeping infants between two and eight hours was $0.1^{\circ} \mathrm{C}$ higher than that of infants sleeping alone $(p<0.04)$. Given the very small variance in rectal temperature this probably reflects a considerable physiological difference between the two groups.

(Arch Dis Child 1996;75:249-250)
\end{abstract}

Keywords: sudden infant death syndrome (SIDS), body temperature, bed sharing.

Recently there has been much interest in the effects on the baby of parent/baby co-sleeping. This has arisen from the hypothesised relations between co-sleeping and sudden infant death syndrome (SIDS). Research in New Zealand has suggested that under certain circumstances co-sleeping may be a risk factor for SIDS $^{1}$ while other researchers suggest the contrary. ${ }^{2}$ It is likely that psychological and physiological links exist between co-sleeping infants and their bed sharing parent(s) and the shared thermal environment is affected. Since temperature has been shown to be linked in some way with SIDS, ${ }^{34}$ we examined the body temperatures of co-sleeping infants and matched controls. Dr S A Petersen,

Department of Pre-Clinica Sciences, Medical Sciences

Building, University of

Leicester, PO Box 138,

Leicester LE1 9HN.

Accepted 30 April 1996

Table 1 Mean values for the non-dichtomous variables of the co-sleeping and control groups. The sleeping position data shows the number of supine $(S)$, lateral $(L)$, prone $(P)$, and mixed (M) positions respectively. Confirmation of the hypothesis that the samples for the co-sleepers are the same as those of the controls is shown by the non-significant $p$ values for each variable

\begin{tabular}{lllllll}
\hline & $\begin{array}{l}\text { Birth weight } \\
(g)\end{array}$ & $\begin{array}{l}\text { Weight } \\
(g)\end{array}$ & $\begin{array}{l}\text { Mean room } \\
\text { temperature } \\
(\mathrm{C} C)\end{array}$ & Tog & $\begin{array}{l}\text { Sleeping } \\
\text { position } \\
S, L, P, M\end{array}$ & $\begin{array}{l}\text { Gender } \\
M, F\end{array}$ \\
\hline Co-sleepers & 3320 & 5090 & 20.3 & 11.9 & $13,8,2,11$ & 21,13 \\
Controls & 3290 & 5090 & 20.7 & 10.6 & $15,5,2,12$ & 20,14 \\
$\mathrm{p}$ & 0.70 & 0.86 & 0.38 & 0.12 & 0.83 & 0.80 \\
\hline
\end{tabular}

Tog $=$ thermal insulation of clothing and bedding.

\section{Methods}

Between 1988 and 1994, over 500 infants were recruited in Leicestershire for overnight recordings of skin and rectal temperatures to study the possible relations between body temperature and SIDS. For each recording, the age, weight, thermal insulation of clothing and bedding (tog), sleeping position, and state of health of the infant were recorded to complement the basic perinatal data. The room, skin, and rectal temperatures were recorded on a Grant Squirrel data logger, sampling at one minute intervals for up to 16 hours. $^{5}$ The infants were studied in their natural environment (the home) and the parents' decision to co-sleep was not affected by the study protocol.

From within the database of recordings, all the infants who regularly slept with one or more parents were identified. Their ages ranged from 6 to 16 weeks. One control for each co-sleeping recording was selected by matching precisely the age, feeding regimens, and parental smoking, and matching as closely as possible the tog level, room temperature, birth weight, weight at recording, sleeping position, and gender. Recordings where the infant was ill or had had an immunisation were not considered for analysis. No recordings were selectively excluded from the data and all bedtime events such as feeding were included.

\section{Results}

Table 1 shows the mean values of the variables which could not be precisely matched for both the co-sleepers and controls. A significance test was performed on each of these variables ( $t$ test for continuous variables and $\chi^{2}$ test for the categorical variables of sleeping position and gender) and the results which are presented in the table show that, for each variable, there was no significant difference between the co-sleepers and the controls. The 34 co-sleeping recordings were then compared with the 34 control recordings with a two way analysis of variance.

The effect of co-sleeping on the rectal temperature of sleeping infants is shown in fig 1 . The mean rectal temperature was calculated for each 30 minute epoch for each infant and the 34 means were used to calculate the mean and standard error for each epoch shown in the graph. After the initial fall in temperature which occurs at bedtime, the rectal temperature of the co-sleeping infants remained approximately $0.1^{\circ} \mathrm{C}$ higher than in the controls for the remainder of the sleep. The mean rectal temperature for each infant was calculated from the temperature data collected between two and eight hours after bedtime, 


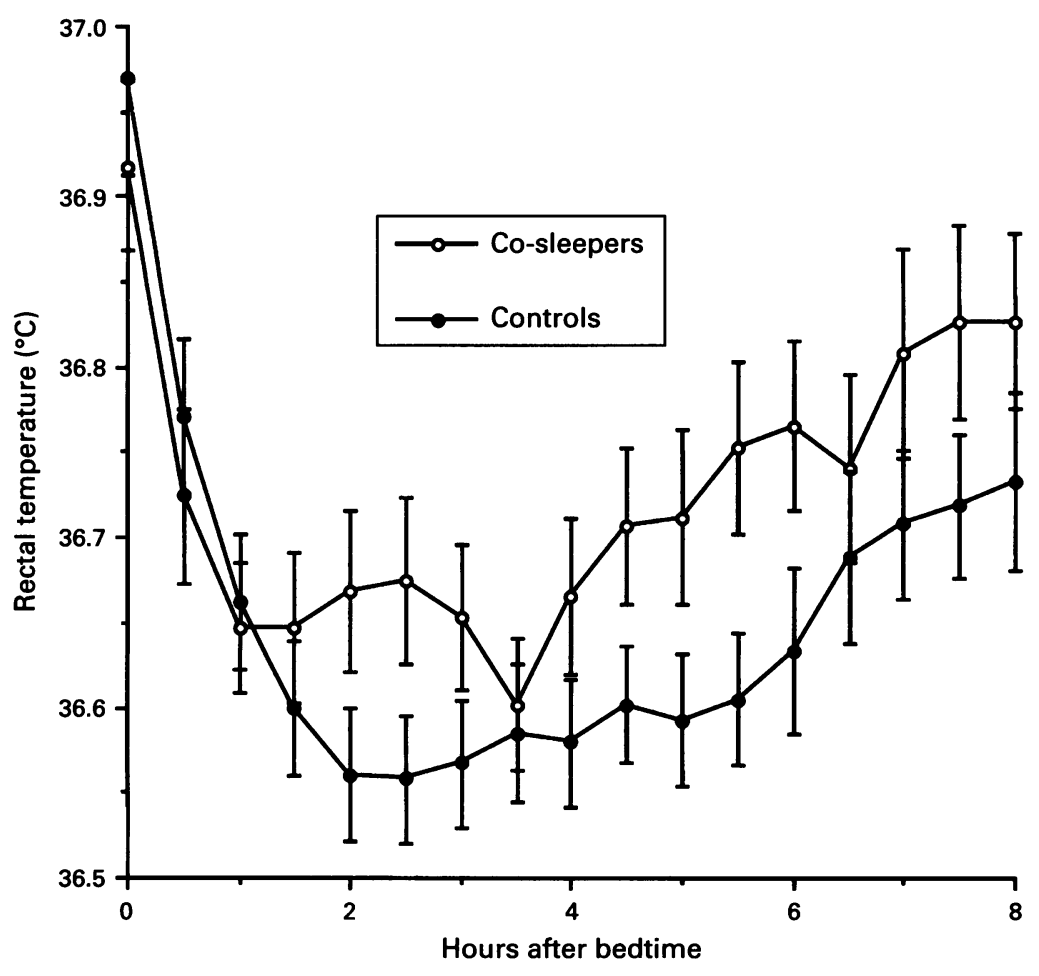

Figure 1 Effect of co-sleeping on the night time rectal temperatures of infants. Data have been averaged across all recordings in the co-sleeping group and also in the control group. The first eight hours of rectal temperatures after the infants have been put to bed is shown. Error bars indicate the standard error.

thus excluding the effect of the initial fall in rectal temperature (co-sleepers $=36.73^{\circ} \mathrm{C}$, controls $\left.=36.62^{\circ} \mathrm{C}\right)$. A two sided Student's $t$ test applied to the mean rectal temperature of each recording yields $p=0.038$, indicating a significant difference between the rectal temperatures of co-sleepers and controls. The average standard deviation of the co-sleeping and control rectal temperatures was $0.277^{\circ} \mathrm{C}$ and $0.241^{\circ} \mathrm{C}$, respectively.

\section{Discussion}

Although the rectal temperature of the cosleeping infants appears to be raised by only a small amount when compared with the controls, the result is statistically significant and may be important. There were no significant differences in any of the variables that were not precisely matched that could clearly account for the increased rectal temperatures of the co-sleeping infants.

The simplest explanation for the higher rectal temperature of the co-sleepers is the thermal coupling between the infant and the parent(s) in the bed. Since the infant is next to another warm body, it is less capable of losing heat, thus increasing the rectal temperature. Although the difference between the tog levels of the co-sleepers and the controls was not statistically significant, a small difference in thermal insulation between these groups might have contributed to the increased rectal temperature in the co-sleepers.

The difference in the rectal temperature of co-sleeping infants perhaps suggests an altered physiological state resulting from the reduced heat loss from the infant. The deep body temperature is regulated at a near constant value by the thermoregulation control system. Therefore, small changes in deep body temperature due to varying thermal loads may indicate large thermoregulation responses. Such changes in thermoregulation almost certainly affect other physiological systems within the body, including the cardiorespiratory system. Some research suggests that the body temperature changes affect sleep state, in contrast with the traditional view that sleep state affects body temperature. ${ }^{6}$ Evaluating the relation between a small but significant increase in an infant's rectal temperature and SIDS is problematical, but these temperature changes may reflect a greater overall physiological effect than that directly measured here.

1 Mitchell EA, Taylor BJ, Ford RPK, et al. Four modifiable and other major risk factors for cot death: the New Zealand study. $\mathcal{F}$ Paediatr Child Health 1992;28(suppl 1):S3-8.

2 McKenna J. Infant-parent co-sleeping in an evolutionary perspective-implications for understanding infant sleep development and the sudden infant death syndrome. Sleep development and

3 Nelson EAS, Taylor BJ. Climatic and social associations with postneonatal mortality rates within New Zealand. NZ with postneonatal mor

4 Ponsonby AL, Dwyer T, Gibbons LE, Cochrane JA, James ME, McCall MJ. Thermal environment and sudden infant death syndrome: case control study. BMF 1992;304:27782.

5 Lodemore MR, Petersen SA, Wailoo MP. Factors affecting the development of night time temperature rhythms. Arch Dis Child 1992;67:1259-61.

6 McGinty D, Szymusiak R. Keeping cool: a hypothesis about the mechanisms and functions of slow-wave sleep. Trends Neurosci 1990;13:480-7. 